\title{
The Soundtrack Of Your Life
}

\author{
Oliver Kierepka, Constantin Brosda, and Christian Geiger \\ University of Applied Sciences Düsseldorf, \\ Josef-Gockeln-Str. 9, 40474 Düsseldorf, Germany \\ \{oliver.kierepka, constantin.brosda, \\ geiger\} afh-duesseldorf.de
}

\begin{abstract}
The rush of nowadays life turned travelling into a means to an end. Commuting people follow the same routes over and over and know less about the places they pass by. In this paper we present our approach of an interactive location-based audio application that invites users to explore alternative routes by sharing location-based song playlists. Therefore a prototypical smartphone application is developed that guides users through existing soundtracks and enables them to share their own playlists, their own Soundtrack Of Your Life.
\end{abstract}

Keywords: Location-based audio, mobile application, interactive multimedia.

\section{Introduction}

Mobility has become a part of our daily life and is more comfortable, less expensive and less exhausting than ever since. Today it is more extrinsic motivated and stronger bound to destinations and timely goals. Instead of interpreting travelling as an experience that offers new perspectives on the world, it often gets reduced to time optimized transportation from one point to another. Some people just know the microcosms of their home place, their workplace and the fastest route between them.

With our project we aim to increase the attractiveness of the surrounding by connecting pedestrian trails and places to an audio experience. The user leaves a virtual trace of the songs one is listening to along the route. This generates what we call the Soundtrack Of Your Life (STOYL).

\section{Related Work}

Tracking solutions enable users to log their individual routes and to share them with a community. Standalone GPS trackers or applications [1] for smartphones with integrated GPS sensors are examples of such services. Other services like Google Latitude [2] use the data to provide real-time information about the current global position of friends. Navigation devices provide assistance based on the current position while travelling to a specified location. Sounds or synthetic voices and graphical user interfaces signal new directions to the user. Warren et al. showed that instead user navigation can be implemented through continuously adapted music [3]. The "Central Park" as a location-aware album extends the idea of a location-based experience by a 
dynamically changing composition depending on the user's current position [4]. The SoundTracking application [5] enables users to share the song-title they are listening to and their current position with others.

\section{Concept}

Listening to music while walking or travelling has become a popular way of entertainment on the go. As we walk around we are able to carry our own individual music library with us. In STOYL the ability of nowadays smartphones is used to increase the appreciation of individual places and to explore the surrounding environment. Therefore real geolocations are linked with song playlists. Each user can leave a virtual track (sound channel) of the songs one listens to while walking. Every channel is unique as it consists of the individual preferences in music and paths. Other users walking along these sound channels are invited to step on new paths besides their well-known ones. As music is able to influence the way we see the world listening to an existing track offers an alternative point of view.

\subsection{User Interaction}

The user is guided through a binaural audio feedback that keeps one on track (see Figure 1). With this audio feedback synthetic voice overlays for navigation are unnecessary. In general the usage of graphical user interfaces is reduced to a minimum to enable the exploration and the visual perception of the surrounding world. Therefore vibro-tactile feedback signalizes other sound channels in proximity.

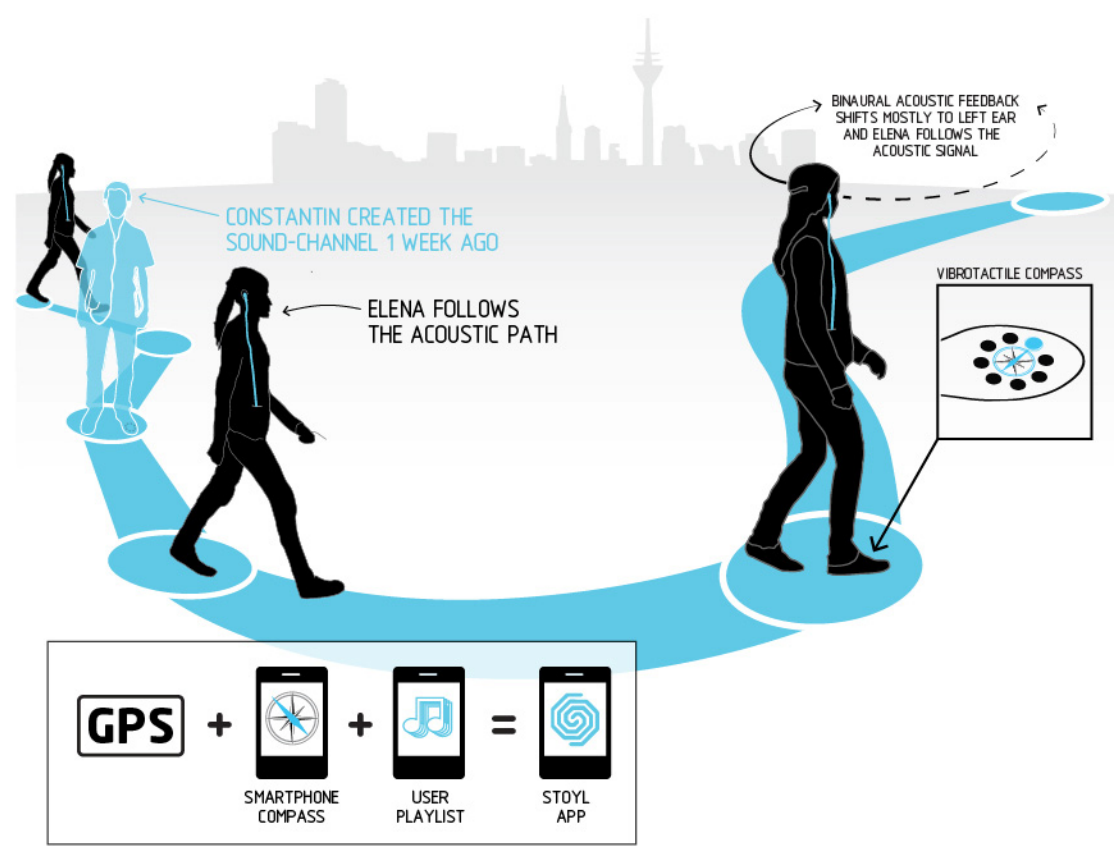

Fig. 1. Sound channel and system 


\subsection{Logging and Exploration}

When in logging mode the application detects the current song the user is listening to. This information is combined with the GPS sensor's data as well as the internal compass. Those sound points are logged periodically and transferred to a server as a unique sound channel.

In exploration mode the user can listen to existing sound channels. If one approaches an other sound channel the vibration motor signalizes this to the user. Afterwards a short stop in walking starts an audio preview of the other channel and the current song is faded out. The user can now decide to enter the sound channel or to stay on the current track and walk on.

The guidance is based on a balance controlled, binaural mapping. If the user is walking straight ahead towards the next point of the sound channel, the current file is played on the right and the left earplug balanced. This rebuilds the perception of real sound sources depending on the angle between direction and the direct way to the source. To ensure a smooth directional navigation the sound channel's positions are interpolated and then used for the directional calculation. If a user walks outside of the channel one is smoothly guided to the next point.

\subsection{Application}

The smartphone is the central element of the application. It manages all incoming data that are gathered by the sensors. Based on this information the current position and direction is calculated and either logged or compared to existing sound channels. Additionally the visual interface and the internal vibration motor are controlled. For audio output a pair of headphones is used that is plugged directly into the smartphone. A web server provides storage and manages all logged sound channels. It can be accessed by the smartphone through standard network connections. For testing all audio files are hosted on the server and streamed to the clients on demand. The system has been implemented as a prototypical smartphone application.

\section{Conclusion}

We introduced the STOYL project that enables users to explore the world in relation to an individual musical playlist. It invites them to explore the environment and thereby to have a closer look besides their well-known paths. This is meant to broaden the user's horizon in two dimensions: first by the different music one is listening to and by the different routes one follows. In future work the prototypical application will be extended and evaluated as a system of multi user interaction.

\section{References}

1. Open GPS Tracker, http: //code.google.com/p/open-gpstracker/ (accessed March 15, 2012) 
2. Google Latitude, http://www.google.com/intl/de/mobile/latitude/ (accessed March 15, 2012)

3. Warren, N., Jones, M., Jones, S., Bainbridge, D.: Navigation via continuously adapted music. In: CHI 2005: CHI 2005 Extended Abstracts on Human Factors in Computing Systems, pp. 1849-1852. ACM, New York (2005)

4. Bluebrain, CentralPark, http://bluebrainmusic.blogspot.de/2011/07/ blog-post.html (accessed Mrz 18, 2012)

5. Soundtracking, http: //www. soundtracking. com (accessed March 13, 2012) 